\title{
Contribution Des Femmes A La Production Piscicole En Côte D'Ivoire
}

\author{
Anoumou Hortense Yao \\ Unité de Formation et de Recherches en Biosciences \\ Université Félix Houphouët-Boigny, Abidjan, Côte d'Ivoire \\ Ahou Rachel Koumi, PhD \\ Centre de Recherches Océanologiques, Abidjan, Côte d’Ivoire \\ Boua Célestin Atsé, PhD \\ Centre de Recherches Océanologiques, Abidjan, Côte d'Ivoire \\ Essetchi Paul Kouamelan, PhD \\ Unité de Formation et de Recherches en Biosciences \\ Université Félix Houphouët-Boigny, Abidjan, Côte d’Ivoire
}

doi: 10.19044/esj.2016.v12n19p325 URL:http://dx.doi.org/10.19044/esj.2016.v12n19p325

\begin{abstract}
Women play a major role in the accession to food security because of their significant contribution to agriculture. However, very few data exists on women's contribution to the Cote d'Ivoire fish production. The objective of this study was to evaluate the ability of fish farms owned by women to contribute to the fish production in Cote d'Ivoire. A survey has been conducted on women fish farms characteristics and their production capacity. Only $5.98 \%$ of 301 farms belonged to women with a contribution to the total production of $2.89 \%$. They are mostly farmers $(44.44 \%)$ aged 30 to 60 years (88.89\%), native of the regions (66.67\%) where they had their farms. Tilapia and catfish are the main species produced with a semi-intensive (83.33\%) farming system in pond. The area of production is less than 1 hectare (77.78\%). Commercial or farms-made feeds are preferably used as fish feeds. Women-owned farms production is characterized by the tilapia commercial weigh over $300 \mathrm{~g}(66.67 \%)$ and a yield of $1000 \mathrm{~kg} / \mathrm{ha} /$ year (77.78\%). By their choice of production system, feeds and their production capacity, women can contribute to set up a sustainable production environment of farm fish in Cote d'Ivoire.
\end{abstract}

Keywords: Fish farming, women, production, contribution, Cote d'Ivoire 


\section{Résumé}

Les femmes jouent un rôle majeur dans l'accession à la sécurité alimentaire en raison de leur importante contribution à l'agriculture. Cependant, très peu de données existent sur la contribution des femmes à la production de poissons en Côte d'Ivoire. L'objectif de cette étude était d'évaluer la capacité des fermes piscicoles appartenant aux femmes à contribuer à la production de poisson en Côte d'Ivoire. Une enquête a été menée sur les caractéristiques des fermes et les capacités de productions des femmes. Seulement 5,98\% sur un total de 301 fermes de production appartenaient à des femmes. Leur contribution à la production piscicole totale est de 2,89\%. Elles sont pour la plupart des agricultrices $(44,44 \%)$ âgées de 30 à 60 ans $(88,89 \%)$, originaires de leur région de production (66,67\%). Les principales espèces élevées sont le tilapia et les silures. Elles pratiquent en majorité le système d'élevage semi intensif (83,33\%) en étang. Les superficies en eau exploitées sont en général inférieures à 1 hectare (77,78\%). Les aliments commerciaux ou fabriqués à la ferme sont de préférence utilisés pour l'alimentation des poissons. La production des fermes appartenant aux femmes est caractérisée par un poids marchand tilapia de plus de $300 \mathrm{~g}(66,67 \%)$ et un rendement supérieur à $1000 \mathrm{~kg} / \mathrm{ha} / \mathrm{an}$ (77,78\%). Par leur choix de système de production, d'alimentation, les femmes peuvent accroître durablement la capacité de production piscicole en Côte d'Ivoire.

Mots clés : Pisciculture, femmes, production, contribution, Côte d'Ivoire

\section{Introduction}

L'aquaculture joue un rôle essentiel dans la sécurité alimentaire des pays développés et en développement (FAO, 2014a). En effet elle contribue à plus de $50 \%$ à la fourniture de poisson sur le marché mondial. La consommation mondiale apparente de poisson est estimée à 19,2 kg/hab./an (FAO, 2014a). Aussi, le développement de l'aquaculture augmente t'il la disponibilité en poisson pour la population. L'aquaculture, contribue à la création d'emploi, à la réduction de la pauvreté et à la promotion de la sécurité alimentaire (Hishamunda et al., 2011). En Côte d'Ivoire, la pisciculture représente l'essentiel de l'activité aquacole (FAO, 2008a). Aujourd'hui, l'on dénombre plus de 1000 fermes piscicoles en Côte d'Ivoire pour une superficie totale exploitée d'environ 750 ha (FAO, 2008a, MIPARH, 2014). La production aquacole nationale de 3720 tonnes est faible et représente 4,69\% de la production halieutique nationale estimée à 79378 tonnes (FAO, 2014b). Cette production nationale couvre seulement 26,46 à $31,75 \%$ des besoins en poisson de la population ivoirienne estimé à 250000 300000 tonnes/an (FAO, 2008a; FAOCI-RAFW 2009). Cette faible 
production halieutique nationale traduit une difficulté d'approvisionnement du marché en protéines animales. Toutefois, avec l'épuisement des stocks halieutiques provoqué par la surexploitation, le développement et la vulgarisation de la pisciculture dans les pays en voie de développement reste la principale option pour satisfaire la demande nationale en poissons (FAO, 2014a).

A l'échelle mondiale, les femmes représentent $43 \%$ des agriculteurs avec une contribution à la main-d'œuvre agricole supérieure à $60 \%$ dans la plupart des pays de l'Afrique subsaharienne (FAO, 2011a). Elles produisent ainsi dans le secteur de l'agriculture et de l'élevage une grande partie de la nourriture de la planète. En Côte d'Ivoire, environ, 67\% de la main d'œuvre féminine travaille dans l'agriculture de subsistance (Leadafricaines, 2012). $\mathrm{Au}$ total, $45 \%$ des femmes agricultrices travaillent dans le domaine des maraîchères, avec un intérêt croissant pour les exploitations agricoles de plus de 10 ha de cultures de café, cacao, ananas, banane, hévéa, palmier à huile, manioc, maïs, igname, les légumes et les élevages de poissons, bovins, ovins, volailles et porcs. Elles sont retrouvées dans le rôle d'encadrement, d'opératrice privée individuelle ou en groupement féminin très actif. Leur effectif et leur potentiel de production en pisciculture reste très peu connus. En effet, après plus de 60 années de pratique piscicole en Côte d'Ivoire, très peu de travaux se focalisent sur la contribution des femmes à la pisciculture. L'objectif de cette étude était d'évaluer la capacité des fermes piscicoles appartenant aux femmes à contribuer à la production de poisson en Côte d'Ivoire. L'étude présente le niveau de gestion, d'intensification et de production de ces fermes afin de déterminer leur potentielle contribution à l'amélioration de la production aquacole nationale.

\section{Matériel et méthodes}

\section{Enquête auprès des pisciculteurs}

Une enquête a été réalisée à l'aide d'un questionnaire et des fiches de renseignements dans les quinze principales régions piscicoles de la Côte d'Ivoire auprès de 301 pisciculteurs de mai à novembre 2013. Le questionnaire a été élaboré avec le logiciel Sphinx 4.5. Les données relevées ont concernées les caractéristiques socio-économiques des promoteurs, les caractéristiques des fermes et les données de production. Les Régions visitées sont celles de la Marahoué, du Haut Sassandra, du Tonkpi, du Cavally, de la Nawa, du Gôh, des Grands Ponts, du Sud Comoé, du District d'Abidjan, de l'Agnéby Tiassa, du Gbeké, du Bélier, du Moronou, l'Indénié Djuablin et de la Mé. Ces Régions ont été identifiées comme étant les principales zones de production piscicole de la Côte d'Ivoire selon les données de l'Association Nationale des Aquaculteurs de Côte d'Ivoire (l'ANAQUACI), de la Direction de l'Aquaculture et des Pêches (DAP) du 
Ministère des Ressources Animales et Halieutique (MIRAH) et de la FAO. L'enquête a consisté à des interviews directes, à la visite des fermes avec le receuil des données de production. Les enquêtes ont été menées chez les pisciculteurs en activité dans les Régions visitées. Les directions régionales ou départementales du Ministère des Ressources Animales et Halieutiques (MIRAH), les Responsables de l'aquaculture à l'Agence Nationale d'Appui au Développement Rural (ANADER), les Responsables locaux de l'ANAQUACI et les associations locales de pisciculteurs ont aidé à la localisation et à l'identification des pisciculteurs et de leur ferme. Au total 301 pisciculteurs ont été enquêtés et visités. Le questionnaire élaboré a permis de renseigner l'origine la tranche d'âge et la fonction principale du promoteur, la localisation des fermes piscicoles, leur durée d'existence, les superficies en eau exploitées, les structures d'élevage, les systèmes pratiqués, les espèces élevées, les différents types d'aliments utilisés, le mode de gestion des fermes et le nombre de personnes travaillant sur les fermes. Ainsi, l'acception des systèmes semi intensif ou extensif de pisciculture a t'elle été faite selon les descriptions de Lacroix (2004) et Lazard (2009).

Les fiches de renseignements ont permis de relever la durée moyenne du cycle de production de poissons marchands, le poids moyen marchands tilapia et la production annuelle.

\section{Traitement des données}

Le rendement des fermes a été calculé selon la formule suivante : Rendement $(\mathrm{kg} / \mathrm{ha} / \mathrm{an})$ = Production annuelle / Superficie en eau exploitée.

Les données concernant essentiellement les fermes piscicoles appartenant aux femmes ont été traitées à partir du logiciel Sphinx 4.5 et présentées sous forme de pourcentage.

\section{Résultats et discussion \\ Résultats \\ Proportion de fermes appartenant aux femmes}

Les données de l'enquête ont montré que seulement 5,98\% des fermes piscicoles enquêtées appartenaient aux femmes (Figue 1). Les promotrices sont rencontrées dans 10 régions sur les 15 visitées. Dans ces Régions, les fréquences de femmes varient entre 2,10 (Mé) et 33,30\% (Tonkpi) (Figure 2). Il n’y avait pas de promotrices sur les fermes visitées dans les régions du Gôh, des Grands Ponts, du Gbeké et du Moronou. Elles sont plus représentées dans les régions du Tonkpi (33,30\%), du Cavally (25,00\%), du Bélier (20,00\%), de l’Agnéby Tiassa (18,20\%) et du District d'Abidjan (14,30\%). 


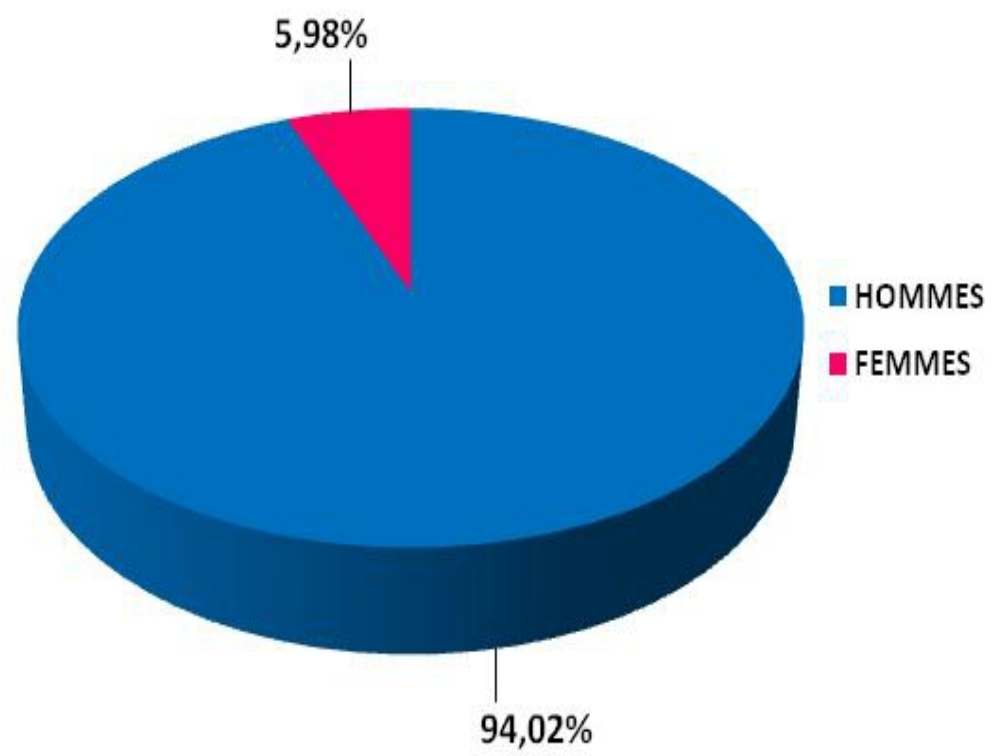

Figure 1 : Répartition des promoteurs de fermes piscicoles en fonction du genre

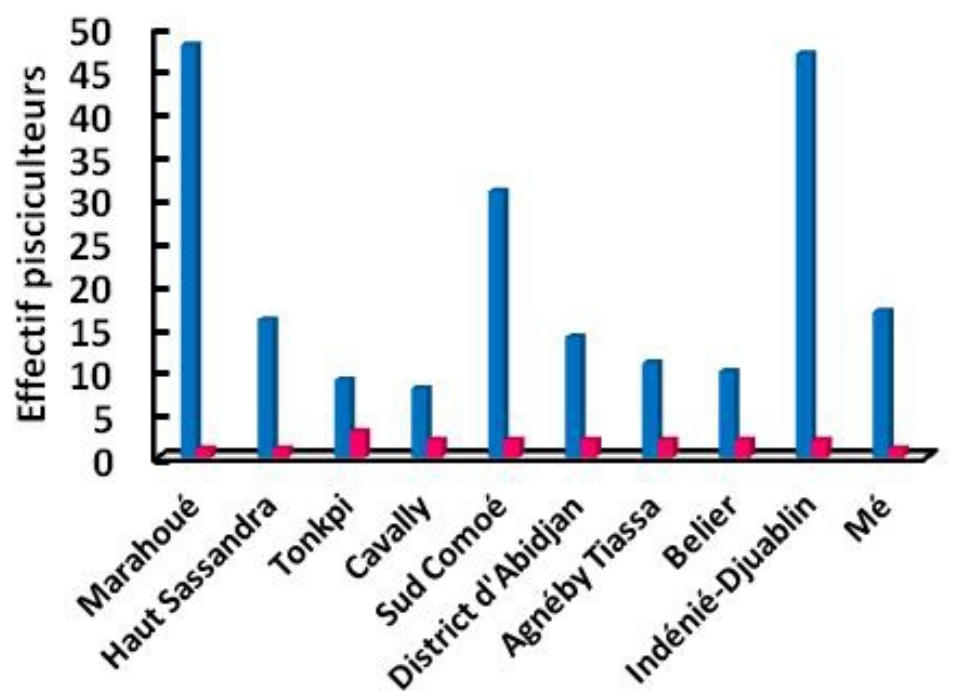

Effectif total pisciculteurs

Effectif pisciculteurs femme

\section{Régions}

Figure 2 : Répartition par région des promoteurs de fermes en fonction du genre 


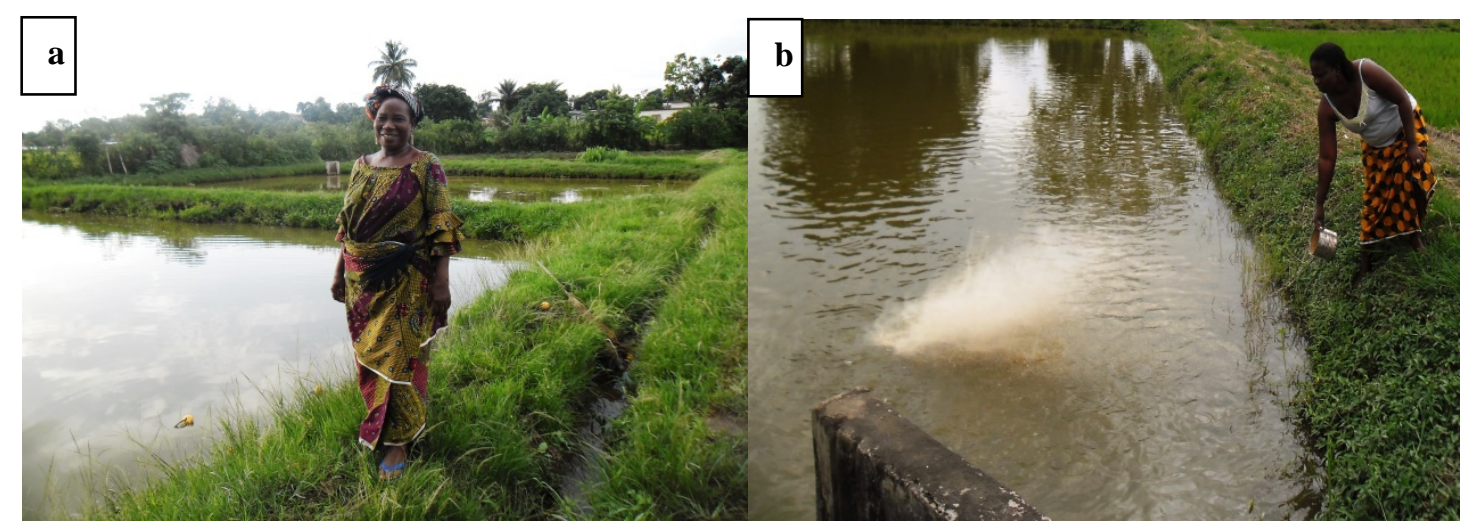

Figure 3 : Femmes pisciculteurs à Danané (a) dans la Région du Tonkpi et à Guiglo (b) dans la Région du Cavally
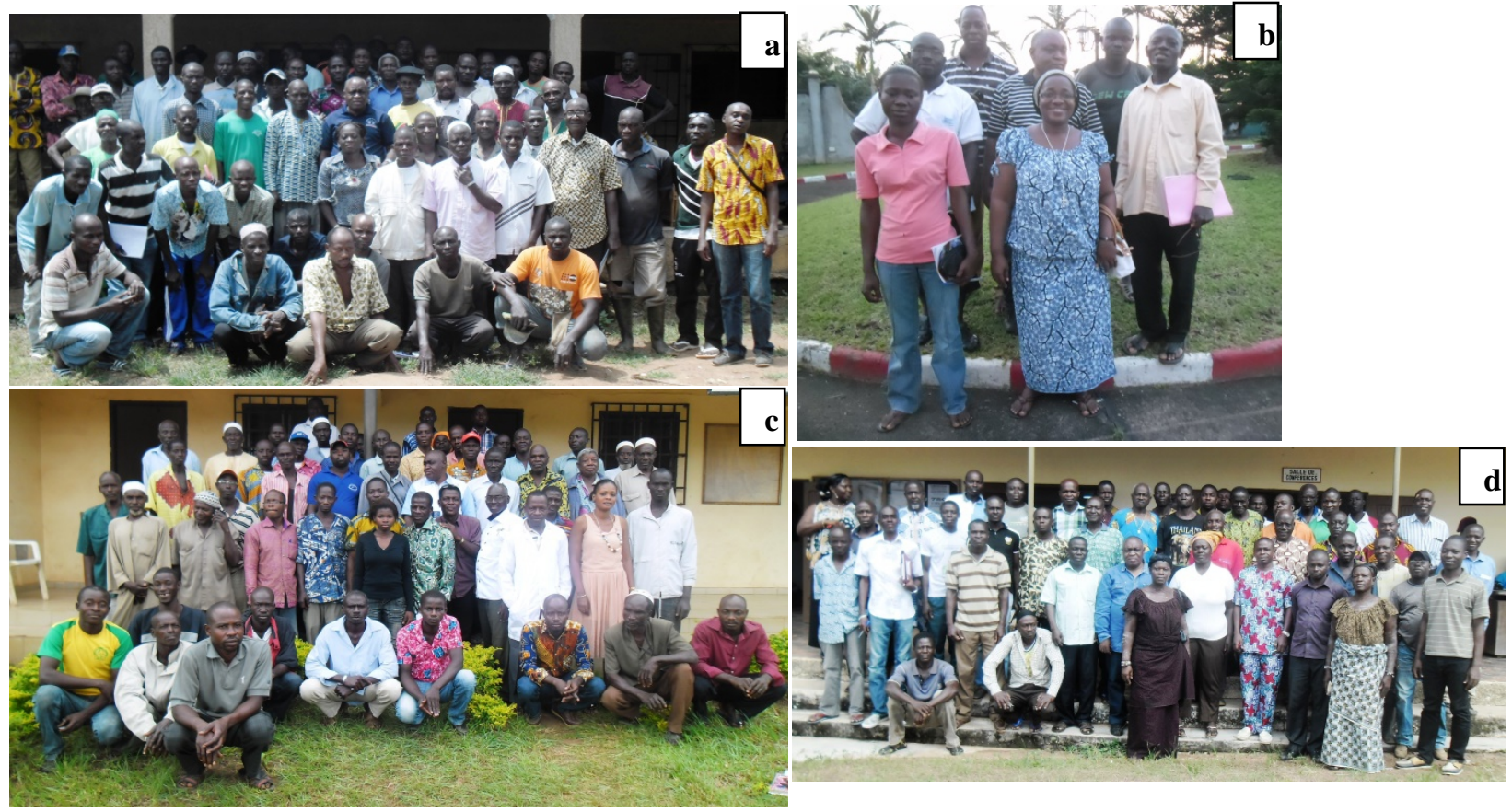

Figure 3 : Promoteurs de fermes piscicoles constitués majoritairement d'hommes dans les Régions du Gôh (a), de la Mé (b), de la Marahoué (c) et de l’Indénié Djuablin (d)

\section{Caractéristiques socio-économiques des promotrices de fermes piscicoles}

Les promotrices de fermes piscicoles sont essentiellement de nationalité ivoirienne (Tableau 1). Elles sont en majorité originaires (66,67\%) de leur région de production. Les tranches d'âge les plus représentatives sont comprises entre $40-50$ ans (33,33\%) et 50-60 ans (27,78\%). Les promotrices agricultrices $(44,44 \%)$ sont les plus nombreuses suivies des salariées $(27,78 \%)$ et des piscicultrices de métier (16,67\%). Les opératrices économiques $(11,11 \%)$ sont faiblement représentées. 
Tableau 1 : Caractéristiques socio-économiques des promotrices de fermes piscicoles

\begin{tabular}{|l|l|l|}
\hline Paramètres & Fréquence & Pourcentage \\
\hline Origine du promoteur & 12 & 66,67 \\
\hline Autochtone & 6 & 33,33 \\
\hline Allochtone & 0 & 0 \\
\hline Allogène & 5 & 27,78 \\
\hline Tranche d'âge de la promotrice (ans) & 33,33 \\
\hline $30-40$ & 6 & 27,78 \\
\hline $40-50$ & 5 & 11,11 \\
\hline $50-60$ & 2 & 27,78 \\
\hline Plus de 60 & 5 & 11,11 \\
\hline Fonction principale de la promotrice & 44,44 \\
\hline Salariée & 5 & 16,67 \\
\hline Opératrice économique & 2 & \\
\hline Agricultrice & 8 &
\end{tabular}

\section{Profil des fermes piscicoles appartenant aux femmes}

Les caractéristiques des fermes piscicoles appartenant aux femmes sont présentées dans le Tableau 2. Ces fermes sont situées à 55,56\% en zone urbaine et périurbaine contre $44,44 \%$ en zone rurale. Le nombre d'années d'existence de la ferme est inférieur à 5 ans pour 33,33\% et compris entre 5 et 10 ans pour 38,89\%. La superficie exploitée de ces fermes est inférieure à 1 hectare pour 77,78\%. Les étangs (100\%) et les étangs-barrages (16,67\%) sont les structures rencontrées sur ces fermes. Le système de production est essentiellement semi intensif (83,33\%). Le tilapia Oreochromis niloticus est élevé sur la totalité des fermes (100\%), viennent ensuite les silures pour 33,33\% des fermes. Les femmes promotrices utilisent les aliments commerciaux $(55,56 \%)$ ou fabriquent leurs propres aliments $(44,44 \%)$ pour nourrir les poissons. Cependant, le tiers d'entre elles (33,33\%) associe les sous-produits agricoles aux aliments commerciaux ou fabriqués. La majorité (72,22\%) des promotrices de fermes piscicoles embauchent un gérant et 55,56\% d'entre elles emploient un personnel composé de deux à quatre agents.

\section{Production des fermes piscicoles appartenant aux femmes}

Le profil de production des fermes piscicoles appartenant aux femmes est présenté dans le Tableau 3. La durée de production de poissons marchands est supérieure à 9 mois pour 72,22\% dans les fermes. Les poids marchands tilapia sont en majorité supérieurs à 300g. La production annuelle de poissons marchands est supérieure à une tonne pour 66,67\% des fermes. Le rendement obtenu est compris entre 1000 et $10000 \mathrm{~kg} / \mathrm{ha} / \mathrm{an}$ pour 66,67\% et plus de $10000 \mathrm{~kg} / \mathrm{ha} / \mathrm{an}$ pour 11,11\%. En somme, la totalité des fermes appartenant aux femmes enregistrent une contribution de 2,89\% à la 
production totale de poissons marchands enregistrée sur les 301 fermes piscicoles enquêtées (Figure 4).

Tableau 2 : Caractéristiques des fermes piscicoles appartenant aux femmes

\begin{tabular}{|c|c|c|}
\hline Paramètres & Fréquence & Pourcentage \\
\hline \multicolumn{3}{|l|}{ Localisation de la ferme } \\
\hline Urbaine et périurbaine & 10 & 55,56 \\
\hline Rurale & 8 & 44,44 \\
\hline \multicolumn{3}{|c|}{ Nombre d'années d'existence (ans) } \\
\hline Moins de 5 & 6 & 33,33 \\
\hline $5-10$ & 7 & 38,89 \\
\hline $10-15$ & 1 & 5,56 \\
\hline $15-20$ & 3 & 16,67 \\
\hline 20 et plus & 1 & 5,56 \\
\hline \multicolumn{3}{|l|}{ Superficie en eau exploitée (ha) } \\
\hline Moins d'un ha & 14 & 77,78 \\
\hline $1-2$ & 2 & 11,11 \\
\hline Plus de 2 & 2 & 11,11 \\
\hline \multicolumn{3}{|c|}{ Structures d'élevage utilisé( ${ }^{(1)}$} \\
\hline Etangs & 18 & 100 \\
\hline Etangs barrage & 3 & 16,67 \\
\hline \multicolumn{3}{|l|}{ Systèmes d’élevage pratiqués } \\
\hline Extensif & 3 & 16,67 \\
\hline Semi intensif & 15 & 83,33 \\
\hline \multicolumn{3}{|l|}{ 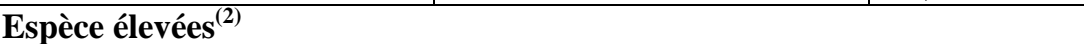 } \\
\hline Tilapia & 18 & 100 \\
\hline Silure & 6 & 33,33 \\
\hline Heterotis & 3 & 9,09 \\
\hline Mâchoiron & 1 & 5,56 \\
\hline \multicolumn{3}{|l|}{ Aliments utilisés ${ }^{(3)}$} \\
\hline Commerciaux & 10 & 55,56 \\
\hline Fabriqués à la ferme & 8 & 44,44 \\
\hline Sous produits agricoles & 6 & 33,33 \\
\hline Aliments non conventionnels & 2 & 11,11 \\
\hline \multicolumn{3}{|c|}{ La promotrice gère sa ferme } \\
\hline Oui & 5 & 27,78 \\
\hline Non & 13 & 72,22 \\
\hline \multicolumn{3}{|c|}{ Nombre de personne travaillant sur la ferme } \\
\hline Moins de 2 & 5 & 27,78 \\
\hline $2-4$ & 10 & 55,56 \\
\hline Plus de 4 & 3 & 16,67 \\
\hline
\end{tabular}

(1) Différentes structures peuvent être présentes sur une ferme

(2) Plusieurs espèces de poissons peuvent être retrouvées sur une ferme

(3) Différents type d'aliment peuvent être utilisés sur une ferme en fonction de l'espèce et du stade de croissance des poissons 
Tableau 3 : Profil de production des fermes piscicoles appartenant aux femmes

\begin{tabular}{|c|c|c|}
\hline Paramètres & Fréquence & Pourcentage \\
\hline \multicolumn{3}{|c|}{ Durée du cycle de production (mois) } \\
\hline Moins de 8,5 & 3 & 16,67 \\
\hline $8,5-9$ & 2 & 11,11 \\
\hline 9 et plus & 13 & 72,22 \\
\hline \multicolumn{3}{|c|}{ Poids moyen du tilapia marchand (g) } \\
\hline Moins de 250 & 1 & 5,56 \\
\hline $250-300$ & 5 & 27,78 \\
\hline 300 et plus & 12 & 66,67 \\
\hline \multicolumn{3}{|c|}{ Production annuelle (kg) } \\
\hline Moins de 500 & 4 & 22,22 \\
\hline 500 à 1000 & 2 & 11,11 \\
\hline 1000 et plus & 12 & 66,67 \\
\hline \multicolumn{3}{|c|}{ Rendements (kg/ha/an) } \\
\hline Moins de 500 & 1 & 5,56 \\
\hline $500-1000$ & 3 & 16,67 \\
\hline $1000-10000$ & 12 & 66,67 \\
\hline 10000 et plus & 2 & 11,11 \\
\hline
\end{tabular}

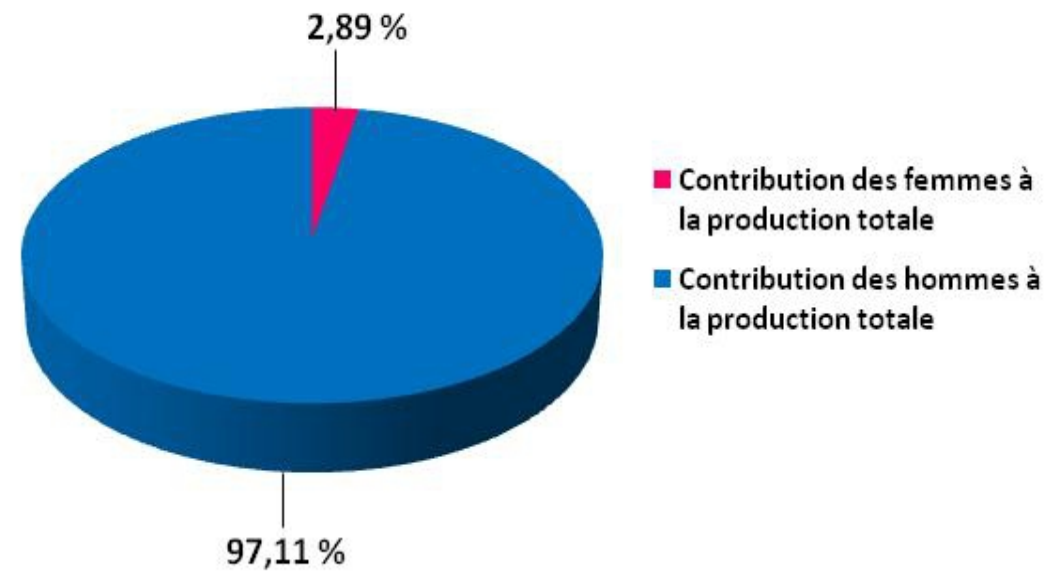

Figure 4 : Contribution des femmes et des hommes à la production de poisson sur les fermes enquêtées

\section{Discussion}

La proportion de femmes (5,98\%) promotrices de fermes piscicoles est faible. La pisciculture en Côte d'Ivoire est donc une activité exclusivement masculine. A l'échelle mondiale, il existe très peu d'information sur le genre dans la pratique de la pisciculture (FAO, 2012). FAO (2008b et 2008c) rapporte 5\% de promotrice de fermes piscicoles au Ghana, 6-10\% au Cameroun. Selon FAO (2012), En Jamaïque, la proportion est substantiellement élevée (8-11\%). Cette faible présence de femmes dans 
la pisciculture serait due à des contraintes sociétales communes aux femmes parmi lesquelles l'accès à la terre, à l'eau, aux moyens de gestion (alphabétisation), au capital (crédit) et les droits d'entreprendre, de disposer de ses revenus et d'investir (FAO, 2011a ; FAO, 2011b; Karacayir et Sacik, 2016). Dans d'autres pays en revanche, la proportion de femmes promotrices piscicoles est relativement élevée. C'est le cas au Nigéria, 20\% et $40 \%$ respectivement dans les Etats de Kaduna et de Lagos. Au Srilanka et au Bangaldesh, les femmes représentent respectivement 30\% et $60 \%$ des pisciculteurs (FAO, 2012 ; Veliu et al., 2009). Ces données suggèrent que dans certaines régions de la Côte d'Ivoire comme dans certains pays, les contraintes liées aux barrières sociétales, aux mœurs et aux coutumes ont pu être atténuées. Les variations observées seraient donc dues à la capacité de certaines régions à se défaire des difficultés liées au genre dans l'agriculture et l'élevage et le niveau de développement de la pisciculture. Cependant, quelques soit le domaine d'activité considéré, Karacayir et Sacik (2016) et Wef (2014) rapportent une dominance du genre masculin dans l'emploi et la productivité agricole sur le genre féminin. La pisciculture n'échappe pas à cette règle dans la majorité des pays du monde (FAO, 2012).

Par ailleurs, les questions de droit foncier, d'accès à la terre et à l'eau justifieraient la forte proportion de productrices autochtones. Les femmes pratiquent en général sur les terres ancestrales. Cette situation explique en partie l'absence totale de promotrices allogènes. Aussi, la proportion de femmes ayant un emploi et salariées traduit-elle l'intérêt de plus en plus croissant des femmes citadines à la pisciculture. L'origine autochtone et le statut salarié représenteraient des atouts pour l'amélioration de la productivité. Selon Idumah (2006) et Oni et al. (2009) la possession de la parcelle exploitée, l'éducation et le statut social influencent positivement la productivité des exploitations agricoles. Les productions enregistrées étant toujours meilleures chez les agriculteurs autochtones, propriétaires terriens et scolarisés. De plus, les femmes enquêtées pratiquent la pisciculture en milieu urbain et périurbaine. La proximité des fermes aux villes traduit une volonté d'investissement, de commercialisation dans les grands marchés à proximité et de gain.

La pratique du système semi intensif de pisciculture en étangs par 83,33\% des femmes contre un total de 51,83\% enregistré au sein de la population enquêtée révèle une réelle volonté managériale et de modernisation de ces femmes. En effet, ce système est pratiqué par excellence dans la plupart des pays à forte production piscicole de l'Amérique, de l'Asie et de l'Afrique tel que l'Egypte, le Nigéria, le Ghana, l'Ouganda et la Zambie (Lazard, 2009). Les rendements enregistrés chez les femmes sont en majorité compris entre 1000 et $10000 \mathrm{~kg} / \mathrm{ha} /$ an et sont dans la gamme de rendements rapportés (1,5 à 15t/ha/an) par Lazard (2009), 
Lacroix (2004) et Mikosalek et al. (2009) en système semi intensif. Le système semi intensif, la monoculture, l'achat d'aliments commerciaux et l'emploi d'un gestionnaire pratiqués par la majorité des piscicultrices témoignent de meilleures conditions de production par rapport à la plupart des pisciculteurs rapportées en Afrique de l'Ouest en générale (Hecht, 2007 ; Gabriel et al., 2007; FAO, 2008a; Crentsil et Ukpong 2014) et particulièrement en Côte d'Ivoire (FAO, 2008a ; Brechbühl, 2009 ; Kimou et al., 2016). Les pratiques observées et les résultats enregistrés, permettent de dire que les femmes pisciculteurs contribuent à la production de poissons marchands de poids conformes aux besoins des marchés de l'Afrique de l'Ouest (Lazard, 2009). Ces résultats sont en accord avec ceux de Odongo et Muhua (2015) qui rapporte qu'en agriculture, la productivité est positivement corrélée au genre féminin, au niveau d’investissement, à la qualité des intrants utilisés et à l'actualisation des techniques. Les piscicultrices ivoiriennes représentent un réel potentiel de production de poissons marchands de qualité en quantité. Cependant, la faible contribution des femmes à la production piscicole rapportée est la conséquence de la faible proportion de femmes enregistrées. Une meilleure implication des femmes à la pisciculture requiert une démarche similaire à celle proposée par la FAO (2011a et 2011b) à savoir la réduction des inégalités entre hommes et femmes, l'accès au crédit, la formation et la vulgarisation des bonnes techniques de production auprès des femmes. De même, la pisciculture en tant que opportunité d'investissement et activité génératrice de revenus et de profit doit être présentée et facilitée aux femmes (GIZ, 2014). Sa promotion à travers les projets spécifiques aux femmes et la création de centre de formation féminin continu en pisciculture aura pour conséquence d'accroitre la proportion de piscicultrices et leur degré d'investissement dans la pisciculture en Côte d'Ivoire.

\section{Conclusion}

La proportion des femmes promotrices piscicoles est faible dans les Régions enquêtées. Cependant, elles présentent un bon niveau d'investissement de production et de rendement. La promotion de la pisciculture auprès des femmes, la définition et la mise en œuvre des mesures d'accompagnements permettraient de susciter leur intérêt et de créer un environnement de production durable de poisson d'élevage en Côte d'Ivoire.

\section{Remerciements}

Les auteurs remercient le Programme d’Appui Stratégique à la Recherche Scientifique (PASRES) pour le soutien financier et expriment leur sincères remerciements aux structures étatiques, aux associations de 
pisciculteurs et à toutes les personnes qui ont facilité la réalisation de cette étude.

\section{References}

Brechbühl, A. (2009). The future of pisciculture in southern Côte d'Ivoire. Bachelor thesis, Federal Institute of Technology - Agri-food and Agrienvironmental Economics Group, Zürich (Swiss), p. 50.

Crentsil, C. \& Ukpong, I. G. (2014). Economics of fish production in Amansie-west District of Ghana: Implication for food security in West Africa. Asian J. Agric. Exten., Economics \& Sociology 3 (3): 179-188.

FAO. (2008a). Fishery and aquaculture country profiles, general view of national fisheries sector the republic of Cote d'Ivoire. Rome, Italie: FAO. ftp://ftp.fao.org/FI/DOCUMENT/fcp/fr/FI_CP_CI.pdf

FAO. (2008b). Fishery and aquaculture country profiles, general view of national fisheries sector the republic of Ghana. Rome, Italie: FAO

FAO. (2008c). Fishery and aquaculture country profiles, general view of national fisheries sector the republic of Cameroun. Rome, Italie : FAO.

FAO. (2011a). Les femmes clé de la sécurité alimentaire. Rome, Italie : FAO au travail.

FAO. (2011b). La situation mondiale de l'alimentation et de l'agriculture. Rome, Italie : FAO.

FAO. (2012). The state of world fisheries and aquaculture. Rome, Italie : FAO.

FAO. (2014a). La situation mondiale des pêches et de l'aquaculture. Possibilité et défis. Rome, Italie : FAO.

FAO. (2014b). Fisheries and aquaculture information and statistics service. Rome, Italie:

http://www.fao.org/figis/servlet/SQServlet?ds=Aquaculture\&k1=COUNTRY \&k1v=1\&k1s=107\&outtype=html (08/11/2014)

FAOCI-RAFW. (2009). Plan Directeur de la Pêche et de l'Aquaculture (PDPA), Rapport de formulation. Abidjan, Côte d'Ivoire : Ministère de la Production Animale et des Ressources Halieutiques.

Gabriel, U. U., Akinrotimi, O. A., Bekibele, D.O., Onunkwo, D.N., \& Anyanwu, P.E. (2007). Locally produced fish feed: potentials for aquaculture development in sub-Saharan Africa. African J. Agric. Research, 2: 287-295.

GIZ. 2014 Genre, pêche et aquaculture. Bonn-Eschborn: Deutsche Gesellschaft für Internationale Zusammenarbeit (GIZ)

Hecht, T. (2007). Review of feeds and fertilizers for sustainable aquaculture development in sub-Saharan Africa. In M. R. Hasan, T. Hecht, S. S. De Silva, \& A. G. J. Tacon (Eds.), Study and analysis of feeds and fertilizers for sustainable aquaculture development (pp. 453- 470). Rome, Italie: FAO Fisheries Technical paper, No. 497. 
Hishamunda, N., Cai, J., \& Leung, P. (2011). Aquaculture commerciale et croissance économique, réduction de la pauvreté et sécurité alimentaire: cadre d'évaluation. Rome, Italie : FAO Document technique sur les pêches et l'aquaculture. No. 512.

Idumah, F.O. (2006). Productivity differentials among food crop farmers in the Niger Delta. PhD thesis, Department of Agricultural Economics, University of Ibadan.

Karacayir, E., \& Sacik, S. Y. (2016). Women's labour in Turkey: A comparasion with selected Oecd countries ${ }^{49}$. European Scientific Journal, ESJ, 12(8).

Kimou, N. B., Koumi, R. A., Koffi, M. K., Atsé, C. B., Ouattara, I. N., \& Kouamé, P. L. (2016). Utilisation des sous-produits agroalimentaires dans l'alimentation des poissons d'élevage en Côte d'Ivoire. Cah. Agric. 25: 25006. DOI: 10.1051/cagri/2016012

Lacroix, E. (2004). Pisciculture en zone tropicale. Hamburg, Allemagne: GTZ, GFA Terra Systems.

Lazard, J. (2009). La pisciculture des tilapias. Cah. Agric. 18: 174-182, pisciculture: le poisson de demain, Synthèse, p. 24.

Leadafricaines. (2012). Egalité hommes-femmes, protection et promotion des droits et de l'émancipation sociale et économique des femmes. Abidjan, Côte d'Ivoire : Care - Union Européenne.

Mikosaleck, O., Barlet, B., Chia, E., Pouomogne, V., \& Tomedi, E. T. M. (2009). Develloppement de la petite pisciculture marchande au Cameroun: La recherche-action en partenariat. Cah. Agric.18: 270-276.

MIRAH. (2014). Plan stratégique pour le développement de l'élevage, la pêche et de l'aquaculture 2014-2020, Abidjan, Côte d'Ivoire: MIRAH.

Odongo, J. A. \& Muhua, G. O. (2015). Modelling the relationship between farmer's attitude towards farming and farm practices: a case study of smallholder farmers in Tanzania. European Scientific Journal ESJ, 11(36).

Oni, O. A., Akinseinde, A. A. \& Adepoju, A. A. (2009). Nonfarm activity and production efficiency of farm household in Egbede local government, Oyo state. Journal of New seeds 10 (1): 1-13.

Veliu, A., Gessese, N., Ragasa C., \& Okali, C. (2009). Gender analysis of aquaculture value chain in northeast Vietnam and Nigeria. Washington DC, USA: Agriculture and Rural Development Discussion Paper 44, World Bank.

WEF (2014). The Global Gender Gap Report. 19.06.2015. http://www3.weforum.org/docs/GGGR14/GGGR_CompleteReport_2014.pd f. 